\title{
PROMOTION OF THE TRANSITION OF ADULT PATIENTS WITH CHILDHOOD- ONSET CHRONIC DISEASES AMONG PAEDIATRICIANS IN JAPAN
}

Yuko Ishizaki *, Masashi Mizuguchi **

* Department of Paediatrics, Kansai Medical University

** Department of Developmental Medical Sciences, Graduate School of Medicine, The University of Tokyo

\section{Background}

$>$ The transition of adult patients with childhood-onset chronic diseases (APCCD) from paediatric to adult healthcare systems is a recent global focus of interest

$>$ As Japan is lagging behind the countries in Europe and North America, the term "transition program" was introduced in Japan in 2006. By 2010, a group of paediatric nurses started a specialized training program aimed at supporting APCCD. In 2013, Japan's Ministry of Health, Labour and Welfare (MHLW) and the Japan Paediatric Society (JPA) proposed specific measures to support the transition of APCCD.

$>$ This study, including the analysis of data retrieved from two databases, attempts to describe the advancement of this concept of healthcare transition in Japan.

\section{Methods}

$>$ To determine the interest of Japanese medical practitioners in the transition of APCCD, the number of English and Japanese articles on the transition of APCCD published in PubMed and the Japan Medical Abstract Society's ICHUSI database was determined using the keywords "childhood-onset chronic diseases" and "transition". Changes in the number of articles and events associated with the promotion of transition were determined by year.

\section{Results}

> Total 129 articles in Japanese and 6 articles in English were retrieved. The number of Japanese-language articles considerably increased after the initiation of the specialized training program for paediatric nurses aimed at supporting patients with APCCD in 2010 and after the MHLW and JPA implemented specific measures to promote transition in 2013.

Number of articles retrieved by childhood-onset chronic diseases and transition

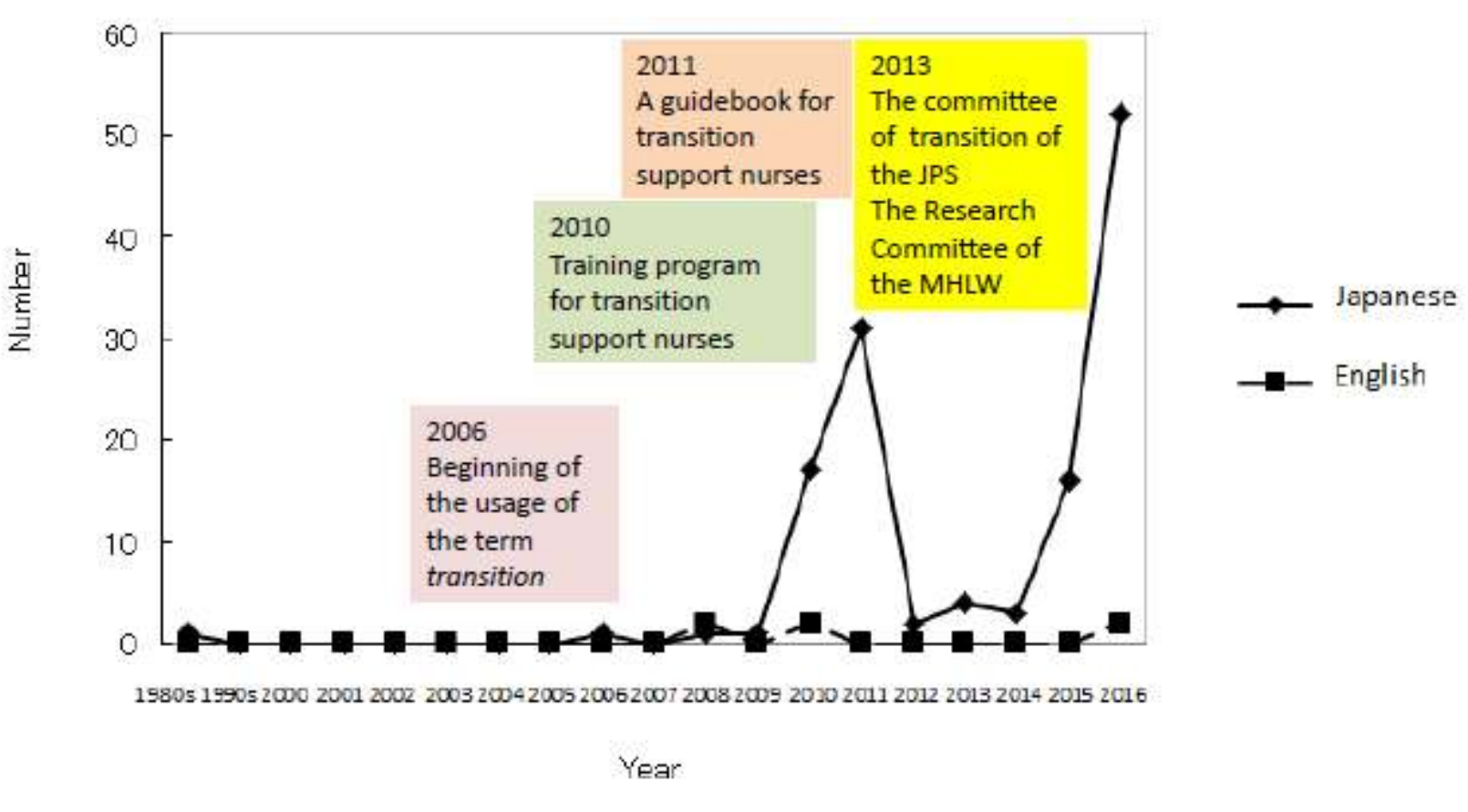

Discussion

> The concept of APCCD healthcare transition increased rapidly over time following the introduction of the MHLW and JPA measures to promote the transition of APCCD.

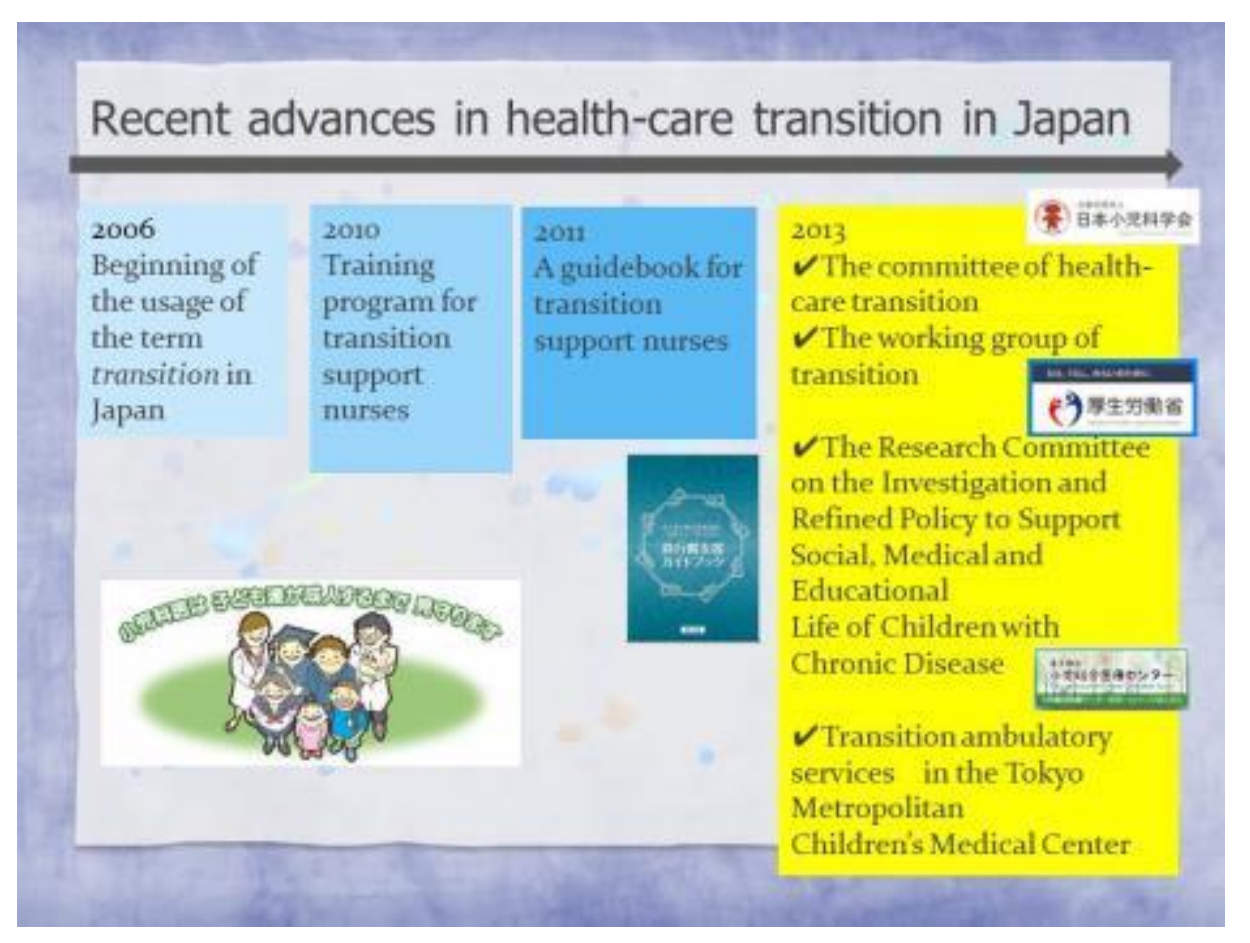

\section{Future directions}

$>$ We plan to construct an information and communications technology (ICT) network linking APCCD with medical facilities to improve their readiness for transition to adult-centered care.

$>$ We aim to develop the Japanese version of the Transition Readiness Assessment Questionnaire (TRAQ) for self-monitoring, which aims to provide selfhelp to patients with chronic conditions, and construct an ICT network to which APCCD can send healthrelated information using mobile phones or computers.

Development of an information and communications technology network model between APCCD and medical practitioners for the improvement of readiness of transition among $A P C C D$

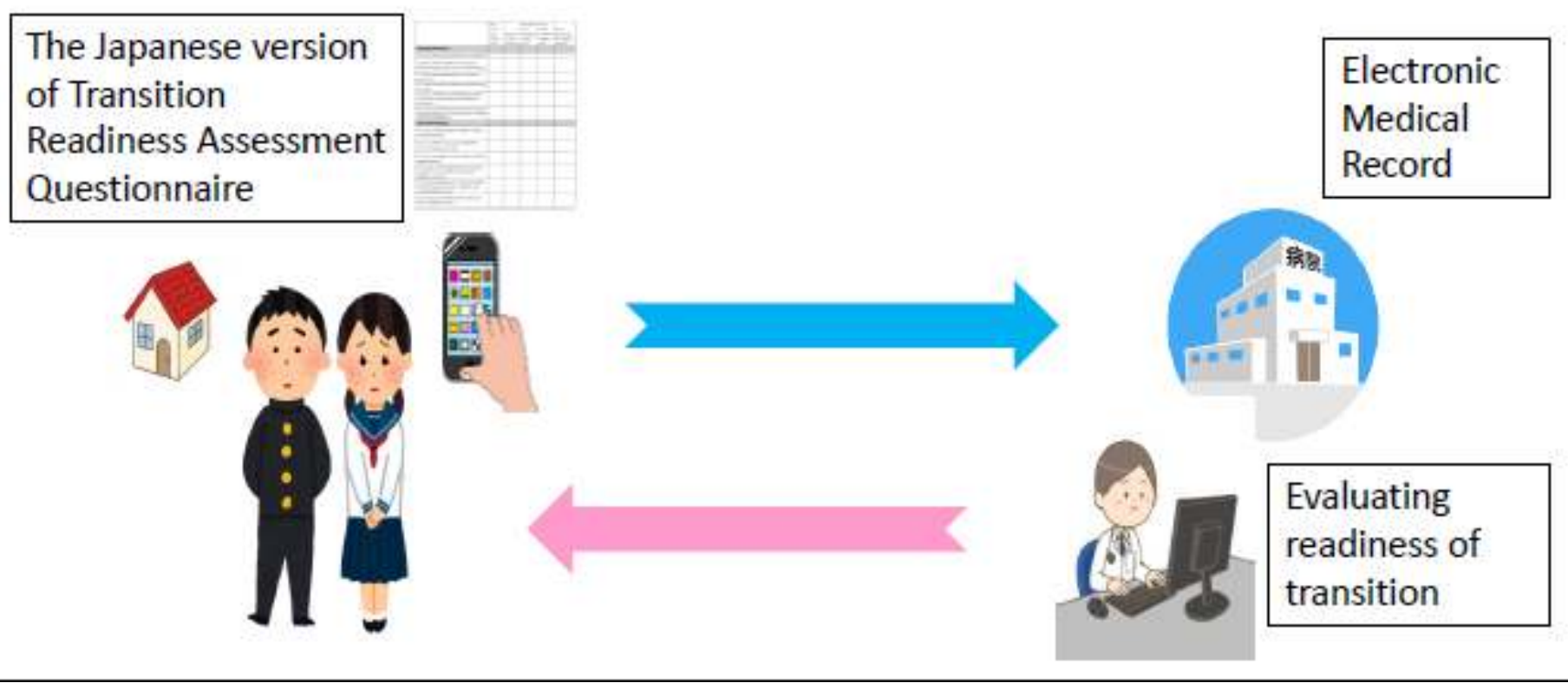

For clinicians: Readiness of transition to adult healthcare is evaluated by the network.

For patients: It is possible to determine a target skill related to self-care behavior which should be acquired in the next step.

Both clinicians and patients can carefully advance the readiness of transition to adult healthcare using TRAQ on the network. 